\title{
Tunnel Point Cloud and BIM Model Integration for Cross-section Monitoring
}

\author{
Wensheng Zhang', Ziqi Hao', Dong Guo², Yingkai Gao² and Jianguo Wang, * \\ 1 School of Traffic and Transportation, Shijiazhuang Tiedao University, Shijiazhuang 050043, China; \\ zws@stdu.edu.cn (W.Z.); haoziqi@stdu.edu.cn (Z.H.) \\ 2 China Railway Shisiju Group Corporation Tunnel Engineering Co., Ltd., Jinan 250000, China; \\ guodong1299@163.com (D.G.); xyns@hotmail.com (Y.G.) \\ 3 School of Civil and Environmental Engineering, University of Technology Sydney, NSW2007, Australia \\ * Correspondence: jianguo.wang@uts.edu.au (J.W.); Tel.: +61-415-552-500
}

\begin{abstract}
This paper introduces a method for tunnel point cloud and BIM model integration and cross-section monitoring, providing information to analyse tunnel cross-sections and surrounding rock deformation, and support for tunnel maintenance and reconstruction. Three types of data are processed for the integration: laser scanning point cloud, BIM tunnel model and terrain model from oblique photogrammetry. An adaptive BIM modelling scheme is proposed for tunnels with alien structures. Precise spatial registration of the data sets is conducted by applying singular value decomposition (SVD) algorithm to calculate transformation parameters from the point cloud model to the BIM model. Since the tunnel central line has high-order derivability, a crosssection calculation method based on tangent vector is proposed to obtain the cross-sectional profile of tunnels at any mileage. The proposed method has been verified by applying it to a tunnel reconstruction project. The experiment results show that the tunnel point cloud and the BIM model were highly coincident after the integration. The developed program can effectively get the cross-section of the tunnel at any mileage, and correctly express the spatial relationship between the BIM tunnel, the point cloud of tunnel and the external mountainous terrain.
\end{abstract}

Keywords: BIM model; point cloud; tunnel engineering; data fusion; cross-section analysis

\section{Introduction}

Building Information Modelling (BIM), as an intelligent 3D model-based digital expression of the physical and functional characteristics of buildings, has the advantages of information integration and sharing, and can provide support for decisions at each stage of their life cycle [1,2]. BIM has been gradually applied to the field of transportation engineering, playing a key role in the engineering design of tunnels, bridges, etc. [3,4]. 3D laser scanning with LiDAR (Light Detection and Ranging) is a non-contact active sensing technology for acquiring dense point clouds of objects surface, and is a popular way for high resolution 3D field surveying [5]. This technology has shown great potential in analysis of the health status of tunnels through cross-section extraction [6,7], quality monitoring [8], and surrounding rock deformation inversion [9-12]. The integration of point cloud and BIM model is called "Scan-vs-BIM" [13], which represents the process of comparing the point cloud of an existing building with its BIM model. It can be used in construction progress observation [14,15], building quality control [16] and full life cycle monitoring [17].

The service performance of a tunnel not only depends on its internal structure, but the external geographical environment which affects the stability of the tunnel structure $[18,19]$, especially for some shallow buried, biased, or soft rock tunnels [20]. Combining the efficiency of BIM and the accuracy of LiDAR surveying to monitor tunnels and surrounding environment through crosssection analysis is a good approach for analysing tunnel service performance and risk avoidance.

A tunnel cross-section profiling method is introduced in this paper, with three types of data collected and processed: laser scanning tunnel point cloud, BIM model and mountain model with oblique photogrammetry. An adaptive modelling scheme for tunnels with alien structures is proposed for the BIM model. Precise spatial registration of the three data set is conducted for their 
integration. Since tunnel central line has high-order derivability, a cross-section calculation method based on tangent vector is proposed. The proposed method has been applied to a tunnel reconstruction project for verification and engineering application.

The rest of the paper is arranged as follows. Sections 2 introduces two types of data collected for the integrated tunnel mode, including LiDAR point clouds and oblique photogrammetry 3D terrain, and generating the BIM tunnel model with parameters. The tunnel model integration with different types of data and the tunnel cross-section analysis is detailed in the Section 3. The validation and engineering application of the proposed method to an existing tunnel is presented in the Section 4, followed by the conclusion as the last section.

\section{Data Collection and BIM Modelling}

\subsection{Laser Scanning Point Cloud Model}

The point cloud tunnel model from 3D laser scanning with LiDAR can represent the real structure inside a tunnel with high precision. Some key sections of the lower part of the mountain model established by the UAV oblique photogrammetry are also selected for 3D laser scanning to enhance the accuracy of the model for the sections.

3D laser scanning technology can gather massive irregular spatial distribution data of 3D point cloud of the internal structure of the tunnel and the surrounding mountain. Each scanning point of the data has a 3D coordinate $(X, Y, Z)$ and other attributes (reflection intensity etc.). Reverse point cloud modelling of tunnels and mountains can be achieved with software processing the information.

\subsection{Terrain Model by UAV Oblique Photogrammetry}

Tunnels are generally built in wide mountain area with complex massif and rock layers. UAV oblique photogrammetry is employed to build a 3D terrain model, with the surface features and rock mass information. Global Positioning System (GPS) and Inertial Measurement Unit (IMU) mounted on the UAV provide the position and attitude information for all the aerial images. In the UAV oblique photogrammetry, the collinear equation is used for precise image alignment.

Mature software and methods are available for UAV oblique photogrammetry [21]. The terrain model established by this technology has the characteristics of complete surface and vivid colours. It is helpful to analyse the surface rock mass morphology of the mountain from a macro perspective. The underlying data structure of the real scene model is a Triangulated Irregular Network (TIN). The optimal image is selected for each triangle of the TIN for mapping, and the terrain model accuracy is limited by the density of the TIN.

\subsection{Parameterized BIM Tunnel Model}

There are many types of tunnel structures. At present, tunnel boring machine (TBM) shielded tunnels have mature automatic parametric modelling scheme as their cross-sections have very good central symmetry [22]. However, tunnels with alien structure still in traditional manual modelling stage. Here we propose a new modelling scheme for alien structure tunnels based on adaptive parametric modelling, and apply it to a straight-wall arched tunnels as an example.

Tunnel road centreline is a very important parameter for BIM model of a tunnel. The shape of road in a tunnel is critical for the safety of vehicles that follow Newton mechanics. Any point on the tunnel road centreline is expected to achieve continuous 2 nd or $3 \mathrm{rd}$ order derivative [23]. As shown in Figure 1, the spline curve is determined by the Hermite interpolation function based on the coordinates of each node P1, P2 etc. The interpolation curve not only strictly passes through each node, but also meet the requirement that the derivatives at each node are continuous $[24,25]$. 


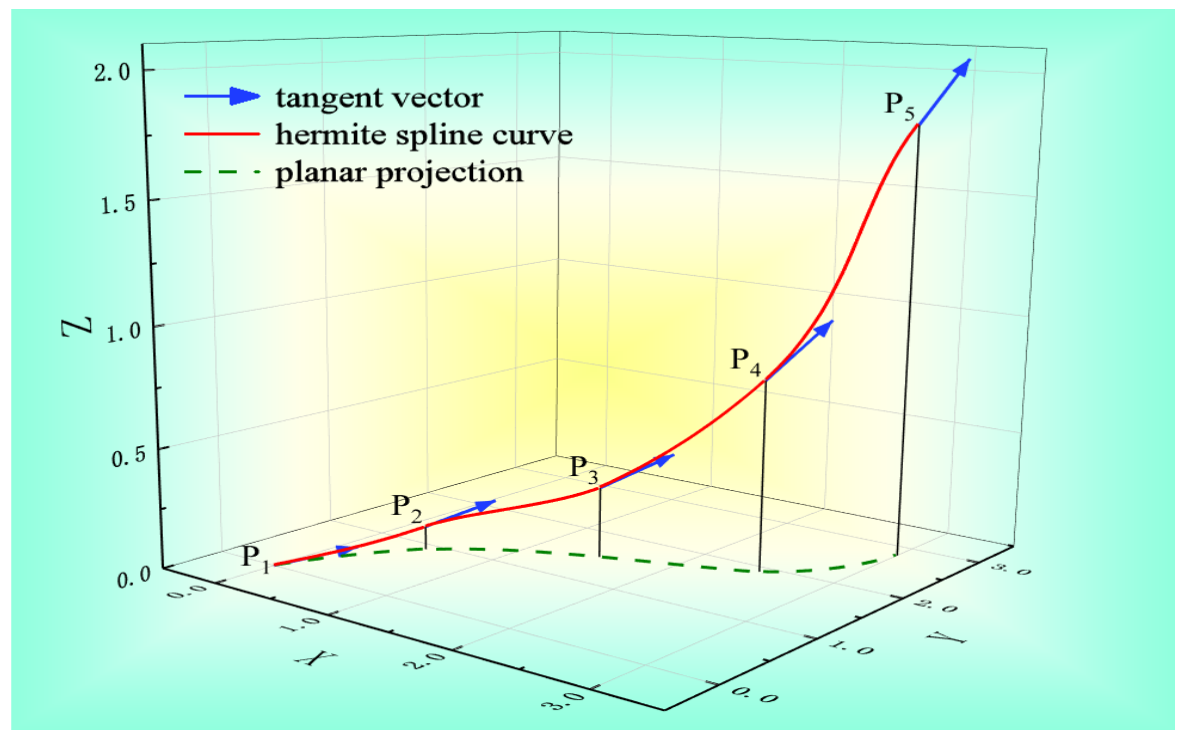

Figure 1. Hermite spline curve as the tunnel road centreline

The conformal characteristics of Hermite interpolation function make it very suitable for tunnel centreline design. According to the line-by-pile coordinates, piecewise two-point cubic Hermite interpolation is used in BIM software to generate a spline curve as the axis of the tunnel.

The basic unit of a straight-wall arched tunnel model is determined based on its geometric parameters. The front and rear cross-sections of a tunnel unit model are positioned at a mileage of the tunnel line. Each tunnel unit model is composed of the upper part and the lower part, and a series of adaptive points are used to control the profiles of the front and rear cross-sections. Figure 2 shows the adaptive points on the upper part (a) and lower part (b) of a tunnel unit model.

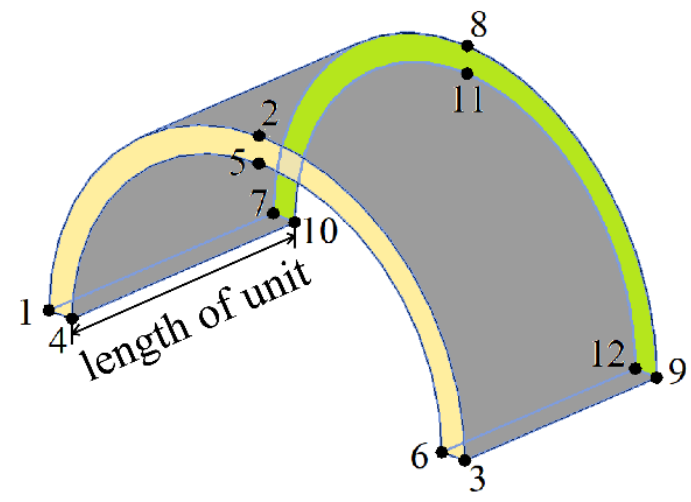

(a)

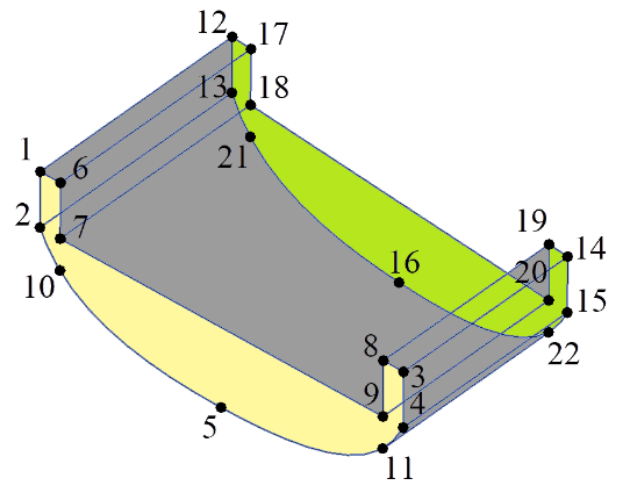

(b)

Figure 2. Tunnel unit model: (a) the upper part and (b) the lower part

The upper part of a unit model is the arch generated by 12 adaptive points as in Figure 2(a), within which the first 6 adaptive points shape the front section of the unit model, the latter 6 adaptive points shape the rear section. The distance between the front and rear sections is defined as the length of a tunnel unit. The lower part including the straight-wall is generated by 22 adaptive points in the similar way as in Figure 2(b).

The design process of the adaptive BIM modelling scheme for alien structure tunnels is shown in Figure 3. The cross-sections are placed in sequence along the tunnel centre line, and then a threedimensional solid model of the tunnel can be established. The tunnel model starts with the first crosssectional profile and ends with the last cross-sectional profile. This method is suitable for variable cross-section tunnel modelling. 


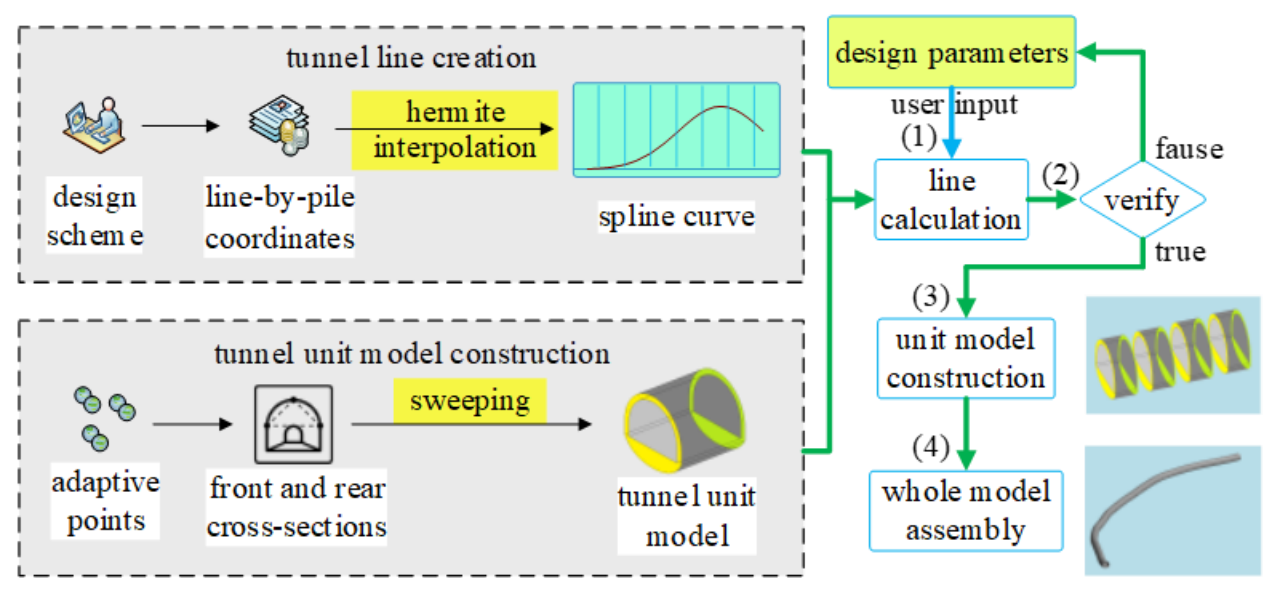

Figure 3. Alien structure tunnels adaptive BIM modelling scheme

As shown in Figure 3, the BIM tunnel modelling process has the following four steps:

1. Information input: enter the tunnel centreline, unit length and other model parameters for data initialization.

2. Centreline segmentation: segment the tunnel along the centreline with the tunnel BIM model unit length. Establish a local coordinate system for each segment; specify the directions of the tunnel as the $\mathrm{Z}$ axis of each local coordinate system; calculate the coordinate of each point on the front and rear cross-section profile, and then conduct the restrictive condition validation.

3. Unit model construction: sweep two cross-sections at adjacent segment points, as the front and rear cross-sections of corresponding mileage, to generate a solid tunnel unit model.

4. Whole model assembly: repeat the step (3) for tunnel unit model construction along the tunnel centreline, and then generate the entire BIM tunnel model.

\section{Tunnel Models Integration and Cross-section Analysis}

The integration of the laser point cloud with the BIM model and the terrain model can fix spatial relationship between them. Then the tunnel cross-section can be used to analyse the relation between the tunnel and its surrounding environment and to monitor the safety of the tunnel.

\subsection{Integration of Point Cloud and BIM Model}

As LiDAR point clouds for the tunnel and lower part of external mountain are surveyed with the same georeferencing system, their relative position is a fixed. However, the tunnel BIM model and the point clouds are generated from different data sources, and need spatial registration for their integration. The spatial registration could be done with iterative closest point (ICP) [26], but it is just suitable to small coordinate difference or an initial estimation available. For large coordinate difference, ICP is inaccurate and slow. Adaptive generic algorithm is another potential method to achieve better accuracy [27], but it is not applicable here as the BIM software we used has no interface for advanced AI algorithms.

Although tunnel point cloud and the BIM model use different surveying data, their geometric characteristics are highly consistent with the same scale, just different in position and orientation. Therefore, a rigid body transformation can achieve accurate spatial registration of the two models. Singular Value Decomposition (SVD) algorithm is accurate and efficient for calculating rigid body transformation matrix between two models [28], and is employed to calculate the rotation and translation parameters in the integration process.

A set of corresponding feature points in the point cloud and the BIM model are required for the spatial registration. As a tunnel is slender and long, the selected feature points should be obvious features evenly distributed in the tunnel, such as features on tunnel vault, entrance, exit, and its road centreline etc. The registration accuracy increases with the number of feature points, but also the computation load. The calculation process of the model registration is as follows: 
1. Solve the central coordinates of the feature point sets of the point cloud and the BIM model:

$$
\begin{aligned}
& J_{S}=\frac{1}{n} \sum_{\mathrm{i}=1}^{N} J_{i} \\
& K_{S}=\frac{1}{n} \sum_{\mathrm{i}=1}^{N} K_{i}
\end{aligned}
$$

where $J_{s}$ is the central coordinate of the point cloud feature point set, $K_{s}$ is the central coordinate of the BIM feature point set. The point cloud feature point set $J_{i}=\left(x_{i}^{(J)}, y_{i}^{(J)}, z_{i}^{(J)}\right)$ is the source data. The BIM feature point set $K_{i}=\left(x_{i}^{(K)}, y_{i}^{(K)}, z_{i}^{(K)}\right)$ is the target data, where $\mathrm{i}=(1,2,3, \ldots, n)$ represents $n$ groups of feature points selected from the two models

2. Decentring the two feature point sets:

$$
\begin{gathered}
\Delta J_{i}=J_{i}-J_{s} \\
\Delta K_{i}=K_{i}-K_{s}
\end{gathered}
$$

where $\Delta J_{i}$ and $\Delta K_{i}$ are the decentralized feature points of point cloud and BIM model respectively. 3. Establish a matrix according to the relative distance between the two new sets of points $H$ :

$$
H=\sum_{\mathrm{i}=1}^{N} \Delta K_{i}^{T} \Delta J_{i}
$$

4. Decompose the metric matrix using the SVD algorithm and obtain a third-order square matrix:

$$
H \stackrel{S V D}{=} U \sum V^{T}
$$

where $U$ and $V$ are unitary matrices of left and right singular vectors respectively, and $\sum$ is a diagonal matrix composed of singular values.

5. Calculate the optimal rotation matrix $R$ and translation vector $t$ :

$$
\begin{aligned}
& R=V U^{T} \\
& t=K_{s}-R J_{S}
\end{aligned}
$$

The coordinates of the point cloud model are adjusted with the rotation matrix $R$ and translation vector $t$ obtained by (7) and (8) after they are imported into the BIM. Then the point cloud model and the BIM model achieve accurate integration with rigid body transformation.

\subsection{Cross-section Calculation Based on Tangent Vector}

Tunnel model data and cross-section can be managed and presented in different ways. Setareh Kokab proposed a slicing algorithm for extracting of cross section profiles from complex point cloud data sets [6]. Wang presented tunnel cross-sectional by rotating tunnel point cloud model in a fixed window to a specific attitude [7].

For the BIM and point cloud integrated model, we proposed a new ross-section calculation based on the tangent vector of any point on the tunnel centreline, as shown in Figure 4 . The cross-section view is at the direction of the tangent vector.

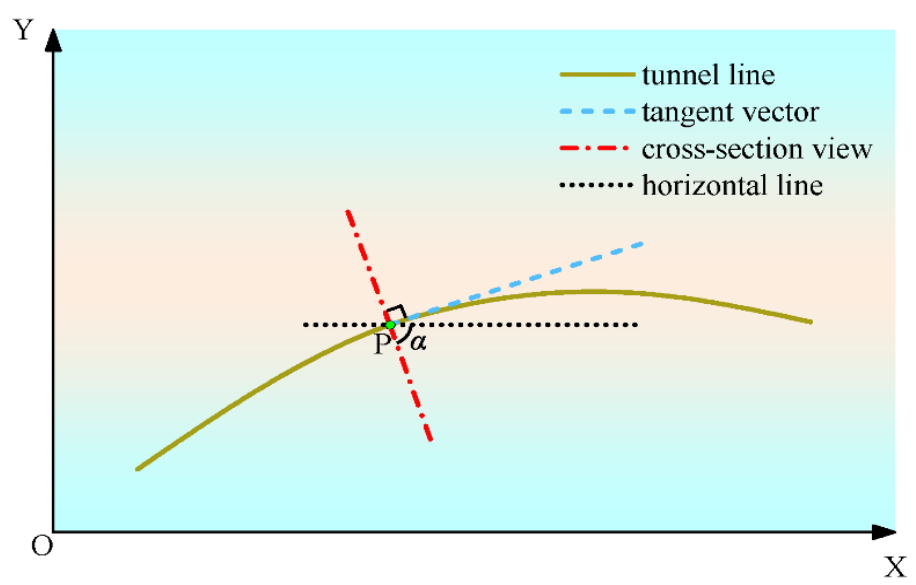

Figure 4. Cross-section calculation based on tangent vector 
This method is easy to be implemented in BIM with simple parameters, and it can be used to calculate the cross-section views for tunnels, highways and bridges which have high-order derivability line structures. It can use the information provide in the integrated tunnel model and be implemented in the BIM software. This provides an efficient way to monitor tunnel cross-sections and the surrounding rock deformation, and decision support for tunnel maintenance and reconstruction. By cutting the integrated model along the direction perpendicular to the tunnel line in the BIM model, cross-sectional views can be obtained. It can be used to analyse the relative position relationship between the point cloud tunnel, external mountain, and the BIM tunnel model. This method utilizes the characteristics of high-order derivability of the tunnel line, and can obtain the cross-sections of the tunnel at any mileage.

\section{Validation and Engineering Application}

The proposed tunnel cross-section monitoring method, based on the integration of point cloud and BIM model, is implemented to an existing tunnel for engineering application exploration.

\subsection{Test Site Background}

The tunnel is situated at a riverbank with undulating terrain of about 200 meters relief. The surrounding rock grades to Category IV. Figure 5 shows the mountain rock images. The tunnel has a straight-wall arched structure with single-layer lining and close to an outer fault cliff with 9 ventilation structures. The tunnel's length is $515 \mathrm{~m}$. The surrounding mountain body subjects to wind and water erosion all year round. The rock layers are severely peeled off, and part of the body has a gravel-like mosaic structure, which may cause risk to the safety of the tunnel.

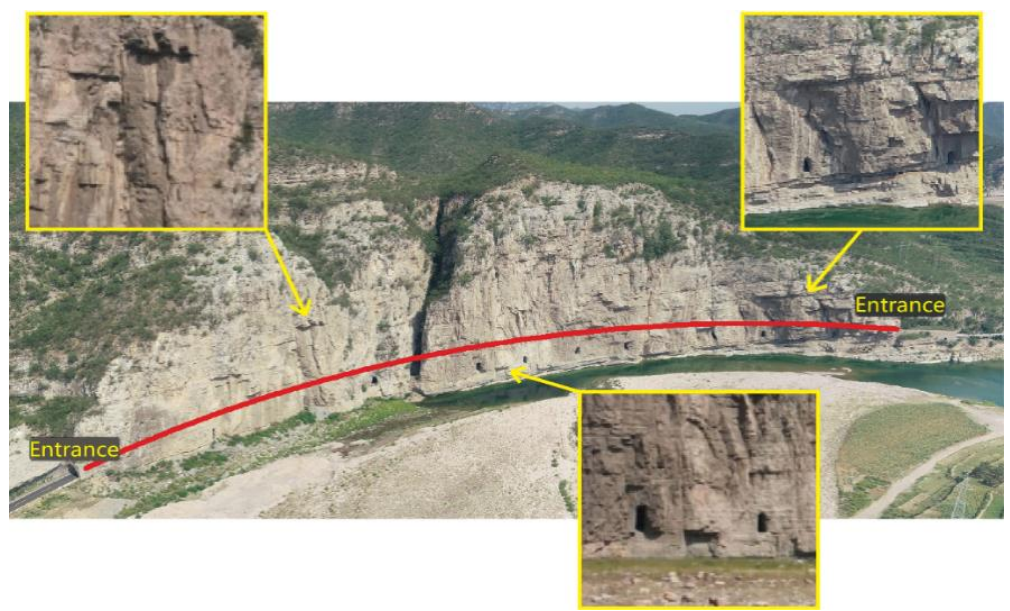

Figure 5. Images of surrounding rock cliff

Autodesk Revit architecture software is the platform for tunnel BIM modelling and point cloud data management. Revit is popular for 3D design of buildings and engineering structures, which supports re-development using the $\mathrm{C \#}$ programming language and has a large user community. The visual programming tool Dynamo in Revit can model complex lines and irregular surfaces. Although the proposed method is applied with Revit, it can also be implemented to other sets of BIM software with suitable functionality.

\subsection{Point Cloud Modelling and Analysis}

The terrain model with the surface features and rock mass information using UAV oblique photogrammetry is presented in Figure $6(\mathrm{a})$. Figure $6(\mathrm{~b})$ is the 3D model of the mountain surface with the location of ground structures and the tunnel entrances. This model can virtually present the surrounding area of the tunnel, with the details of mountain, river, road, and villages etc. It can provide useful information for tunnel design and construction, but not accurate nor detailed enough for analysing the condition of the mountain surface outside the tunnel. 


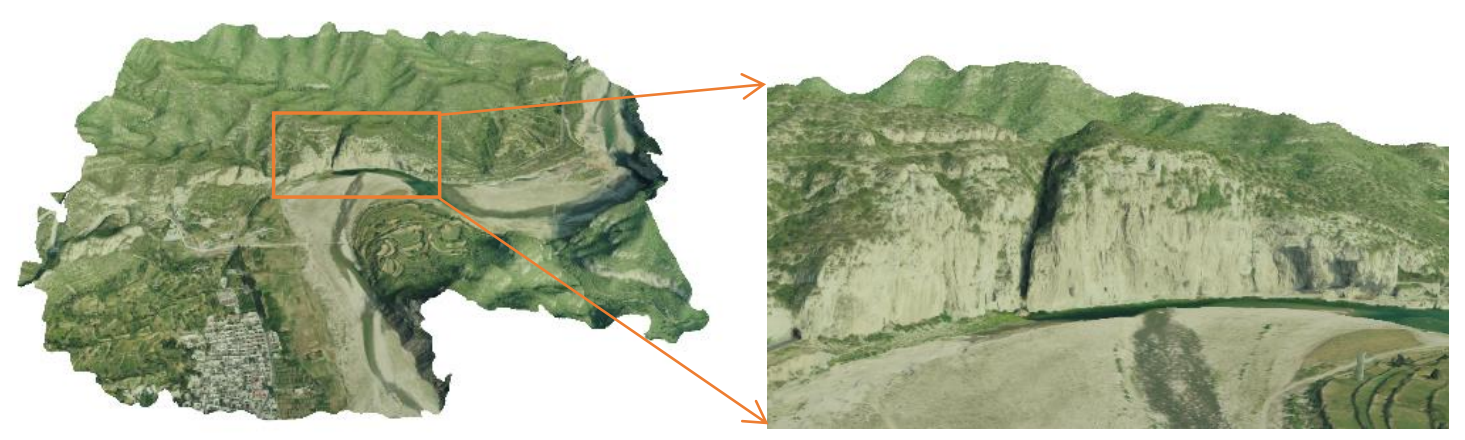

(a)

(b)

Figure 6. Oblique photogrammetry terrain model: (a) entire and (b) mountain surface outside the tunnel

The point cloud of the tunnel and the cliffy mountain collected with LiDAR can represent the structure with high precision. The point cloud data was processed by filtering, denoising, adjustment, registering and splicing etc. The total length of the tunnel is about $515.0 \mathrm{~m}$. The lowest altitude of the cliffy mountain is $315.0 \mathrm{~m}$ and the highest is $475.0 \mathrm{~m}$. Figure $7(\mathrm{a})$ shows the cliffy mountain LiDAR point cloud model, from top to bottom as elevation, colour, and laser reflection intensity. Figure 7(b) depicts two local protruding rock cliff topography, and the opening and the entire body of the straight-wall arched tunnel model.

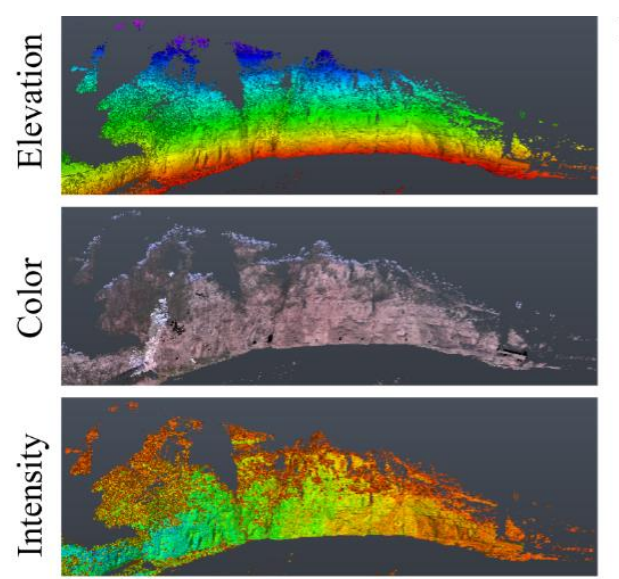

(a)

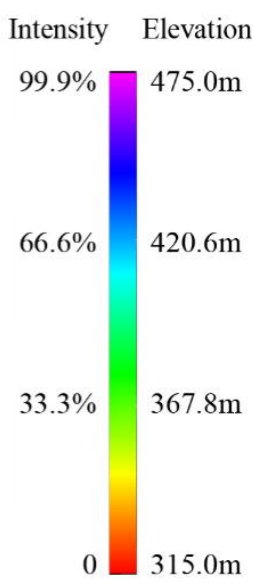

$15.0 \mathrm{~m}$

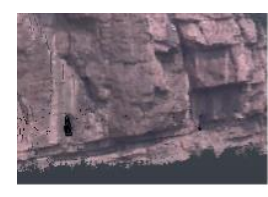

protruding rock 1

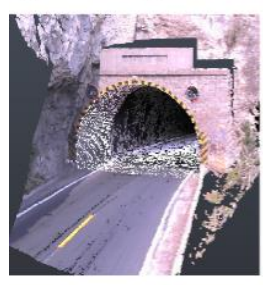

opening

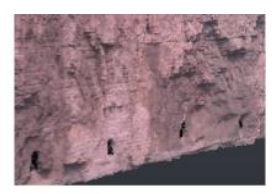

protruding rock 2

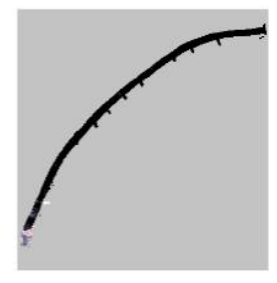

entire body

(b)

Figure 7. Point cloud model of cliffy mountain and tunnel: (a) cliffy mountain analysis and (b) cliffy mountain rock and tunnel

\subsection{BIM Adaptive Modelling}

Based on the proposed adaptive BIM modelling scheme for tunnels with alien structure, Dynamo visual programming workflow was used to develop a tunnel modelling program for parameterization of tunnel centreline line and structure. Tunnel BIM model can be quickly generated after inputting relevant parameters, include tunnel structure unit length $S$; side wall height $\boldsymbol{h}$; outer radius of arch $r$; road width $l$ and lining thickness $\boldsymbol{d}$, as showing in Figure 8.

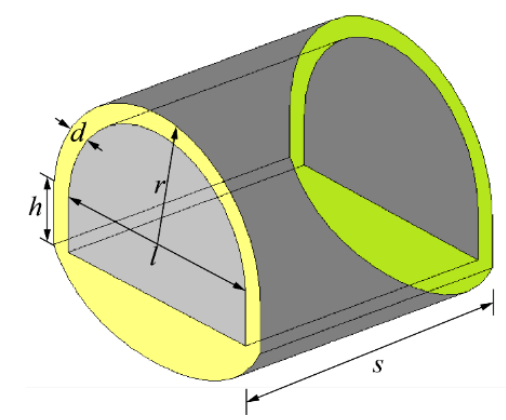

Figure 8. Parametric tunnel structural unit model 
Figure 9 shows the tunnel model generated by the proposed method with a set of parameters, where the tunnel unit length is $5 \mathrm{~m}$, the side wall heights is $1.56 \mathrm{~m}$ a, the outer radii of the arch is $4.2 \mathrm{~m}$ and the lining thicknesses is $0.2 \mathrm{~m}$ respectively. Figure 9 (a) is the entire tunnel model and Figure 9 (b) is a small section of it. The tunnel BIM model follows the tunnel centreline with smooth connection between sections. The total length of the tunnel is $515.0 \mathrm{~m}$, and it took about 20.36 s to generate the model.

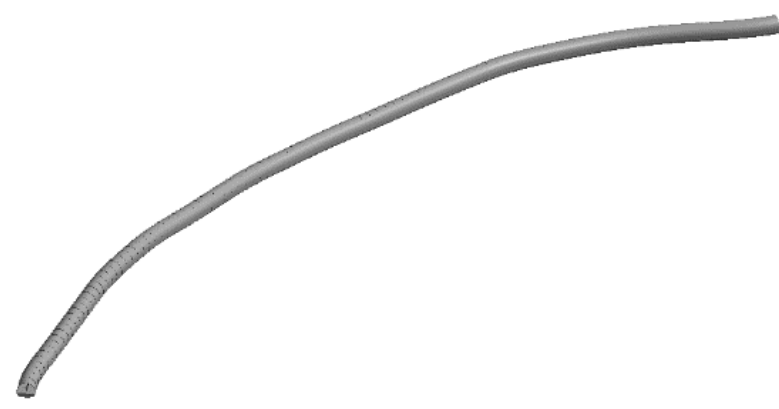

(a)

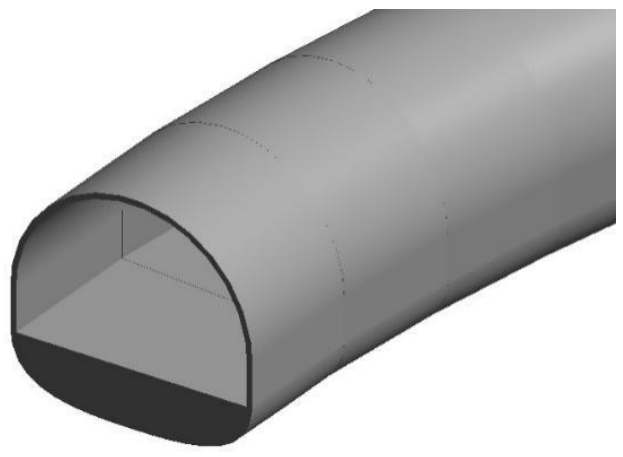

(b)

Figure 9. Tunnel BIM model by adaptive Hermite interpolation: (a) entire tunnel and (b) partial tunnel

\subsection{Model Registration and Integration}

The tunnel point cloud and BIM model are integrated after registration with SVD, as detailed in the Section 3.1. Nine pairs of feature points were selected at the entrance, exit, tunnel centreline, etc. from the point cloud and the BIM model respectively. The feature points of the point cloud were set as the source data and the points of BIM model as the target data. SVD can calculate the optimal rotation matrix $R$ and translation vector $t$, and then use Revit to adjust the posture and orientation of the point cloud. The integrated model is shown in Figure 10, where the point cloud is black and the BIM model is grey. The figures show that the black and grey overlap very well, which indicates that the two models have a high degree of correspondence after the integration.

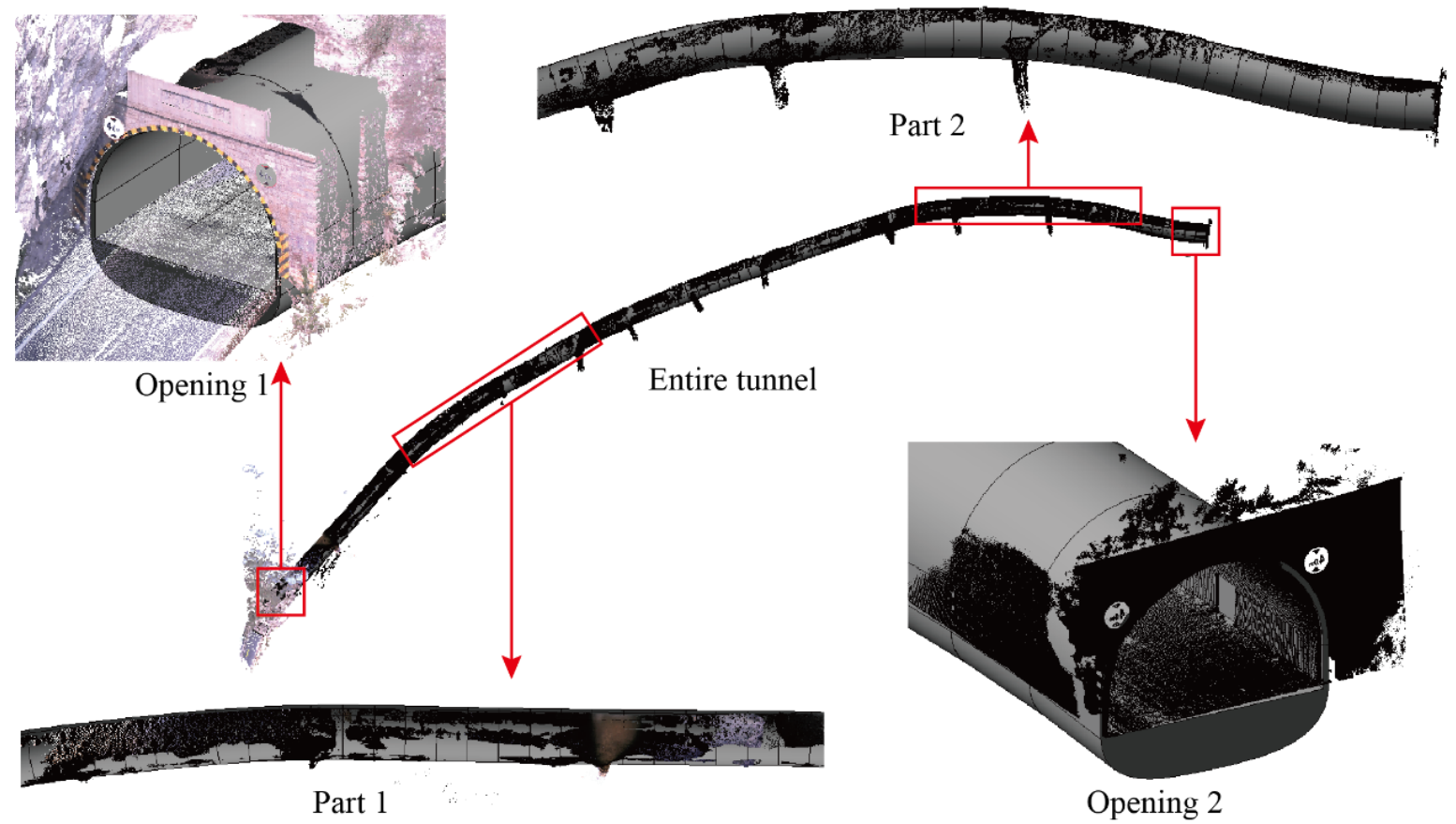

Figure 10. Integration of point cloud and tunnel BIM model 


\subsection{Tunnel Cross-section Display}

Applying the cross-section calculation method described in the Section 3.2, a tunnel cross-section monitoring program was developed with Revit. Figure 11 shows four cross-section views at different mileages. from which we can see the cross-section views correctly express the relationship between the relative positions of the BIM model and the point cloud of the tunnel and the external mountain. The coordinates of the point cloud and the BIM model coincide well in general at all the views, which verifies that the registration using SVD is effective.
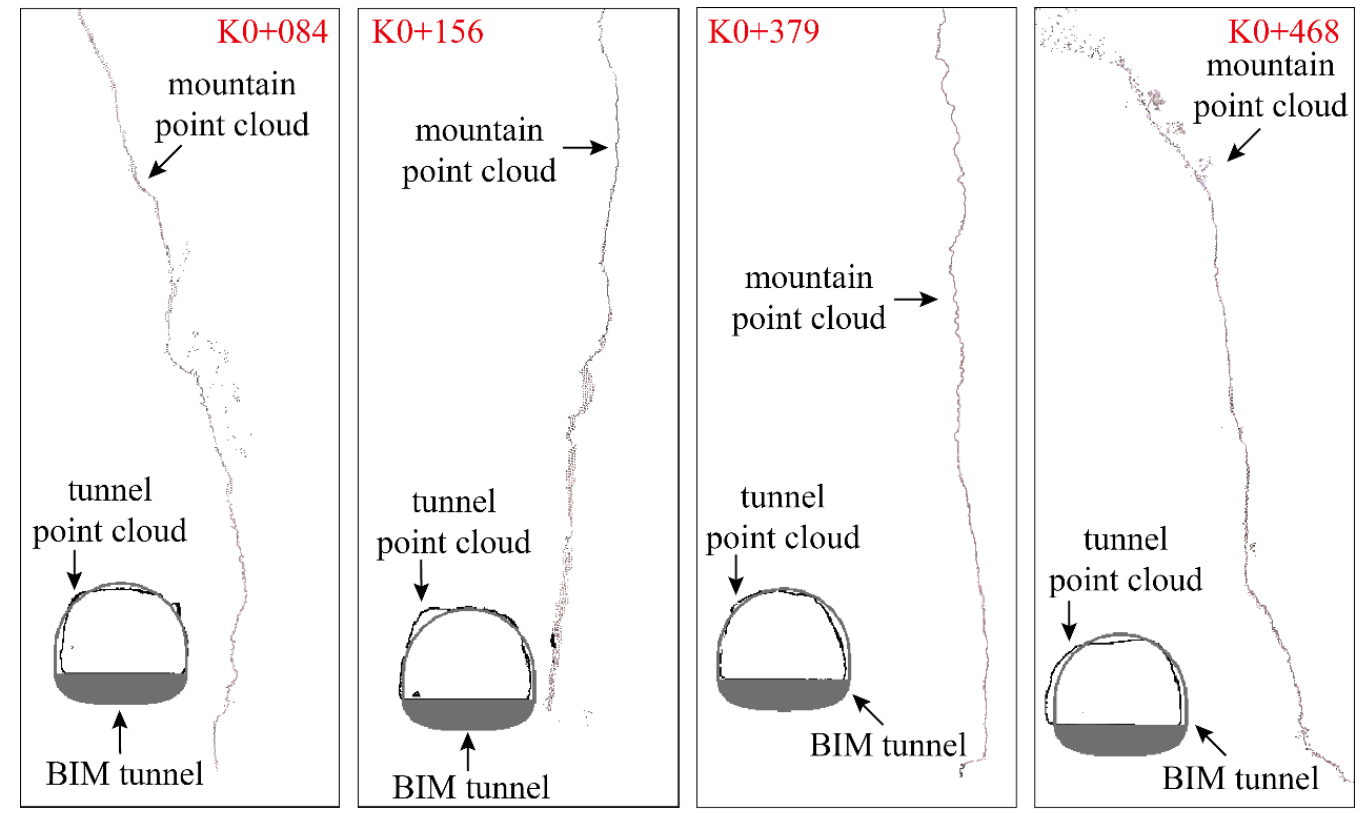

Figure 11. Integration of point cloud and tunnel BIM model

\section{Discussion and Conclusions}

Three types of data are collected and processed for the integrated tunnel model, including LiDAR point cloud, BIM tunnel model and terrain model with oblique photogrammetry. An adaptive modelling scheme for tunnels with alien structures is proposed for the BIM modelling. Precise spatial registration of the three data set is conducted for their integration. SVD algorithm is applied to calculate transformation parameters from the point cloud model to the BIM model, to achieve accurate spatial registration during the integration process.

Since tunnel central line has high-order derivability, a cross-section calculation method based on tangent vector is proposed to obtain the cross-sectional profile of the tunnel at any mileage. This method can obtain the cross-section of the integrated tunnel at any mileage. The cross-sections of the integrated tunnel can correctly express the spatial relationship between the BIM tunnel, point cloud tunnel and external mountain. It can monitor the tunnel cross-sections and surrounding rock deformation to evaluate the service performance of the tunnel.

The proposed method has been applied to a tunnel reconstruction project for verification and engineering application. The results show that the tunnel point cloud and the BIM model are highly coincident after integration. The developed program can get the cross-section of the tunnel at any mileage, and can correctly express the spatial relationship between the BIM tunnel, the point cloud tunnel and the external mountain.

BIM software has a powerful graphics engine and is very efficient to manage the integrated tunnel model and to view tunnel cross-sections. The proposed adaptive BIM modelling scheme for tunnels with alien structure can generate BIM tunnel model according to a set of parameters and a tunnel centreline. It can flexibly adjust the centreline shape and geometric size of the tunnel model with a fast modelling speed. The fineness of the parameterized BIM model can be further improved in the future. 
By analysing the differences between cross-sections of the point cloud and the tunnel model in BIM, it can provide useful information for monitoring tunnel deformation, detecting dangerous tunnel sections, and evaluating the service performance of the tunnel. This tunnel cross-section monitoring method combines LiDAR point cloud with BIM model, and can provide decision support for the reconstruction or expansion of existing tunnels.

Supplementary Materials: Multi panoramic views of the tunnel located area are available online at www.720yun.com/t/2dvkilfhp7m?scene_id= 33065258 .

Author Contributions: Conceptualization, W.Z. and D.G.; methodology, W.Z.; software, Z.H.; validation, Z.H., Y.G. and W.Z.; formal analysis, Y.G.; investigation, Z.H.; resources, D.G.; data curation, Z.H.; writing - original draft preparation, Z.H.; writing-review and editing, W.J.; visualization, Y.G.; supervision, W.Z. and W.J.; project administration, W.Z.; funding acquisition, W.Z. and D.G. All authors have read and agreed to the published version of the manuscript.

Funding: This work was supported by the Science and Technology Development Fund Project (No.206Z0801G), the Overseas Expertise Introduction Project in Hebei Province (2020) and the Key Research and Development Program of Hebei Province (No.18390324D).

Acknowledgments: We thank Senior Engineer Jie Li and his team at the Shijiazhuang Institute of Surveying, Mapping \& Geotechnical Investigation, for surveying and mapping technique support.

Conflicts of Interest: The authors declare no conflict of interest. The funders had no role in the design of the study; in the collection, analyses, or interpretation of data; in the writing of the manuscript, or in the decision to publish the results.

\section{References}

1. Zhang, L.; Chu, Z.W.; Song, H.B. Understanding the relation between BIM application behavior and sustainable construction: a case study in China. Sustainability 2020, 12(1), 306.

2. Wang, M.J.; Qian, Y.M.; Yuan, R.J.; Wang, Q.E. Visual analysis on evolution, hotspots and frontier of international BIM research -Literature metrology based on web of science database. Journal of the China Railway Society 2019, 41(06), 9-15.

3. Kaewunruen, S.; Sresakoolchai, J.; Zhou, Z.H. Sustainability-based lifecycle management for bridge infrastructure using 6D BIM. Sustainability 2020, 12(6), 2436.

4. Cheng, Y.J.; Qiu, W.G.; Duan, D.Y. Automatic creation of as-is building information model from singletrack railway tunnel point clouds. Automation in Construction 2019, 106, 102911.

5. Yang, B.S.; Liang, F.X.; Huang, R.G. Process, challenges and perspectives of 3D LiDAR point cloud processing. Journal of Surveying and Mapping 2017, 46(10), 1509-1516.

6. Kokab, H.S.; Urbanic, R.J. Extracting of cross section profiles from complex point cloud data sets. IFACPapersOnLine 2019, 52(10), 346-351.

7. Wang, W.X.; Chen, W.W.; Wang, K.; Li, S. Extraction of tunnel center line and cross-sections on fractional calculus, 3D invariant moments and best-fit ellipse. Optics \& Laser Technology 2020, 128, 106220.

8. Xu, J.; Ding, L.Y.; Luo, H.B.; Chen, E. J.; Wei, L.C. Near real-time circular tunnel shield segment assembly quality inspection using point cloud data: a case study. Tunnelling and Underground Space Technology 2019, 91, 102998.

9. Jiang, Q.; Zhong, S.; Pan, P.Z.; Shi, Y.N.; Guo, H.G.; Kou, Y.Y. Observe the temporal evolution of deep tunnel's 3D deformation by 3D laser scanning in the Jinchuan No. 2 Mine. Tunnelling and Underground Space Technology 2020, 97, 103237.

10. Zhang, L.S.; Cheng, X.J. Tunnel deformation analysis based on Lidar points. Chinese Journal of Lasers 2018, $45(04), 225-230$.

11. Lee, S.J.; Choi, S.O. Analysing the stability of underground mines using 3D point cloud data and discontinuous numerical analysis. Sustainability 2019, 11(4), 945.

12. Cui, H.; Ren, X.C.; Mao, Q.Z.; Hu, Q.W.; Wang, W. Shield subway tunnel deformation detection based on mobile laser scanning. Automation in Construction 2019, 106, 102889.

13. Bueno, M.; Bosché, F.; González-Jorge, H.; Martínez-Sánchez, J.; Arias, P. 4-Plane congruent sets for automatic registration of as-is 3D point clouds with 3D BIM models. Automation in Construction 2018, 89, 120-134. 
14. Pučko, Z.; Šuman, N.; Rebolj, D. Automated continuous construction progress monitoring using multiple workplace real time 3D scans. Advanced Engineering Informatics 2018, 38, 27-40.

15. Kim, S.; Kim, S.; Lee, D.E. Sustainable application of hybrid point cloud and BIM method for tracking construction progress. Sustainability 2020, 12(10), 4106.

16. Liu, J.D.; Zhang, Q.L.; Zhang, J.H. Construction management and quality control of prefabricated building based on BIM and 3D laser scanning. Journal of Tongji University (Natural Science) 2020, 48(01), 33-41.

17. Ding, Z.K.; Liu, S.; Liao, L.H.; Zhang, L. A digital construction framework integrating building information modelling and reverse engineering technologies for renovation projects. Automation in Construction 2019, $102,45-58$.

18. Darinde, G.; Marian B.R.; Daan, S.; Marcel, H. Integrating sustainability into major infrastructure projects: four perspectives on sustainable tunnel development. Sustainability 2020, 12(1), 6.

19. Zhang, Z.Q.; Gong, R.K.; Zhang H.; He, W.P. The sustainability performance of reinforced concrete structures in tunnel lining induced by long-term coastal environment. Sustainability 2020, 12(10), 3946.

20. Li, C.; Lu, X.P.; Zhu, N.N.; Lu, Y.; Wu, Y.B.; Li, G.Q. Continuously extracting section and deformation analysis for subway tunnel based on LiDAR points. Journal of Surveying and Mapping 2015, 44(09), 10561062.

21. Li, L. 3D road network extraction method based on UAV oblique photography. China Journal of Highway and Transport, 2019,32(11):219-226+254.

22. Zhong, Y.; Chen, J.; Chen, G.L.; Wu, J.M. Shield tunnel structure information modelling method based on building information modelling technology. Rock and Soil Mechanics 2018, 39(05), 1867-1876.

23. Li, M.; Wei, Q.C.; Pan, Z.H.; Zang, C.Z.; Qin, X.C.; Shi, J. Three-dimensional high-order continuous curve alignment design of horizontal and vertical overlapping region of high-speed railways. Journal of Traffic and Transportation Engineering 2018, 18(06), 50-60.

24. Fageot, J.; Aziznejad, S.; Unser, M.; Uhlmann, V. Support and approximation properties of Hermite splines. Journal of Computational and Applied Mathematics, 2020, 368, 112503.

25. Tao, T.Y.; Wang, H. Reduced simulation of the wind field based on Hermite interpolation. Engineering Mechanics, 2017, 34(03), 182-188.

26. Wu, Z.Z.; Chen, H.C.; Du, S.Y. Correntropy based scale ICP algorithm for robust point set registration. Pattern Recognition, 2019,93:14-24.

27. Li, G.Y.; Wang, J.; Liu, X.H. et al. Point cloud and BIM model registration based on improved adaptive generic algorithm. Bulletin of Surveying and Mapping, 2020(02):160-162.

28. Akritas, A. G.; Malaschonok, G. I. Application of singular-value decomposition (SVD). Mathematics and Computers in Simulation, 2004,67(1-2):15-31. 